hep-th/9303113

DFTT 10/93

March 1993

\title{
ANOMALY-INDUCED MAGNETIC SCREENING IN 2+1 DIMENSIONAL QED AT FINITE DENSITY
}

\author{
Stefano Forte \\ I.N.F.N., Sezione di Torino \\ via P. Giuria 1, I-10125 Torino, Italy
}

\begin{abstract}
We show that in $2+1$ dimensional Quantum Electrodynamics an external magnetic field applied to a finite density of massless fermions is screened, due to a $2+1$ dimensional realization of the underlying 2-dimensional axial anomaly of the space components of the electric current. This is shown to imply screening of the magnetic field, i.e., the Meissner effect. We discuss the physical implications of this result.
\end{abstract}

Submitted to: Physical Review Letters 
Quantum Electrodynamics in $2+1$ dimension has attracted recently considerable theoretical attention, due to both theoretical and experimental motivation from condensed matter systems: on the one hand, several startling features of planar quantum mechanics, such as fractional statistics 11 ) and Chern-Simons field theory (2) have started revealing themselves; on the other hand both the Quantum Hall Effect and High- $\mathrm{T}_{c}$ superconductivity are crucially related to the effectively two-dimensional nature of the systems where they have been discovered. Indeed, the possibility of excitations carrying arbitrary statistics, effectively described by coupling of the charged excitations to a Chern-Simons field theory, is at the basis

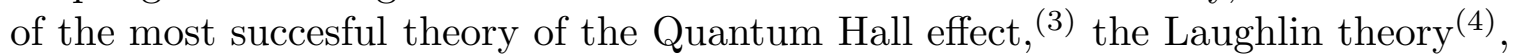
and is perhaps at the root of high $\mathrm{T}_{c}$ superconductivity 5 ).

The appearance of superconducting behavior in $2+1$ dimensional systems with fractional statistics seems to be a rather robust feature of any theory of fermions coupled to Chern-Simons fields (6), at least at zero temperature. Because a ChernSimons interaction is always generated dynamically by quantum fluctuations in the effective action of 2+1-dimensional fermions coupled to external gauge potentials( $($ d), unless forbidden by symmetry, it is quite conceivable that superconductivity induced by the topological structure of the theory which is associated to fractional statistics should be a more general feature of $2+1$-dimensional electrodynamics.

Here, we shall show that this is indeed true in a well-defined sense: in $2+1$ dimensional electrodynamics with a chemical potential, i.e., with finite fermion density, an analogue of the London equation always holds; this (for magnetic fields below a critical value) leads to the Meissner effect, i.e., screening of the magnetic field, which is a hallmark of superconductivity.

Rather than analyzing the effective action of fermions coupled to the gauge potentials, and discussing possible relations of our result to the usual approaches based on an effective Chern-Simons coupling and fractional statistics, we shall establish our result by direct computation of the vacuum expectation value of the electric current and its curl. We shall show that the the electric current, i.e., the space components of the three-dimensional covariant fermionic current of the theory, satisfies, to first order in the magnetic field, a $2+1$-dimensional analogue of the anomaly equation in two dimensions. This gives to its curl an anomalous, nonvanishing vacuum expectation value, proportional to the magnetic field itself, with a coefficient that depends on the parity-breaking parameter, i.e., on the chemical potential. Thus, quantum fluctuations induce anomalous vacuum currents which screen the applied magnetic field, with a screening length depending on the chemical potential. We shall establish this result for time-independent gauge fields, as well as for time dependent ones at the leading order in the adiabatic approximation, in the low temperature limit. We shall also discuss briefly the physical relevance of our results.

We study $2+1$ dimensional massless electrodynamics with a chemical potential term, defined by the Lagrangian

$$
L=\int d^{3} x\left[-\operatorname{tr} \frac{1}{4 e^{2}} F_{\mu \nu} F^{\mu \nu}+\bar{\psi}\left(\gamma^{\mu}\left(i \partial_{\mu}-A_{\mu}\right)-\mu \gamma^{0}\right) \psi\right]
$$


where the coupling $e^{2}$ has the dimensions of a mass. We shall work throughout in the $A_{0}=0$ gauge. Our starting point is the observation that the spatial components of the fermion current in 2+1-dimensional Minkowski space-time are closely related to the axial current of a 2-dimensional Euclidean theory. Indeed, the fermion current of the theory (1) is

$$
j^{\mu}=\bar{\psi} \gamma^{\mu} \psi
$$

where we can represent the 2+1-dimensional Dirac matrices in terms of Pauli matrices as $\gamma^{i}=i \sigma^{i},(i=1,2), \gamma^{0}=\sigma^{3}$. Consider now the usual Dirac $\alpha$ matrices, $\alpha^{i}=\gamma^{0} \gamma^{i}$, which satisfy a two-dimensional Euclidean Clifford algebra $\left\{\alpha^{i}, \alpha^{j}\right\}=\delta^{i j}$, and may thus be used to construct a Euclidean Dirac operator

$$
\begin{aligned}
i \not D & =\left(i \partial_{i}-A_{i}\right) \alpha^{i}-\mu \\
& \equiv-H^{(\mu)}=-(H+\mu)
\end{aligned}
$$

The Dirac operator (3) is readily recognized to coincide with (minus) the Hamiltonian $H^{(\mu)}$ of the given theory (1); notice that the chemical potential term is formally identical to a two-dimensional (imaginary) mass term. Recalling that in a two-dimensional Euclidean theory $\gamma_{5}=i \gamma_{(2)}^{1} \gamma_{(2)}^{2}$, it follows that the axial current associated to the Dirac operator (3) is

$$
\begin{aligned}
j_{5(2)}^{i} & =i \psi^{\dagger} \gamma_{(2)}^{i} \gamma_{5} \psi \\
& =i \psi^{\dagger} \alpha_{i} \sigma_{3} \psi
\end{aligned}
$$

which is related to the spatial components of the $2+1$-dimensional Minkowski current (2) by

$$
j_{5(2)}^{i}=\epsilon^{i j} j^{i}
$$

where $\epsilon^{i j}$ is the completely antisymmetric tensor in two dimension normalized as $\epsilon^{12}=1$.

Now, the current (4) satisfies an axial anomaly equation (8)

$$
\partial_{i} j_{5(2)}^{i}=-\frac{1}{\pi} B(x)
$$

where $B(x)=\epsilon_{i j} \partial^{i} A^{j}(\vec{x})$. We are thus led to conjecture that for time-independent fields, and more generally at the leading order in the adiabatic approximation, the spatial part of the current $j_{(3)}^{\mu}(t, \vec{x})$ at fixed time $t$ should satisfy a similar equation. This Eq. would then have the structure of a London equation. A minute's reflection reveals that the conjecture cannot be literally true, for the two-dimensional current (4) and the three-dimensional one (2) do not have the same dimensions, thus the space components of the latter cannot satisfy Eq.(6) as is. Also, an equation of the form (6) for the space components of the current signals breaking of parity in the $2+1$ dimensional theory (just as the presence of a Chern-Simons term in the action) and can therefore be induced by radiative correction only in the presence of 
a parity-breaking parameter. As we shall see, the presence of a chemical potential will provide both the parity breaking, and the required dimensionful parameter.

We shall now proceed to establish our main result, namely, prove the conjecture that the space components of the current (2) satisfy a two-dimensional anomaly equation of the form (6). To this purpose, we consider the $\zeta$-function regularized (9.10) expression for the vacuum expectation value of the current Eq.(2):

$$
\left\langle j^{\mu}(x)\right\rangle=\lim _{s \rightarrow 1} \operatorname{tr}\left[\gamma^{\mu} R(x, x ; s)\right]
$$

where $R(x, y ; s)$ is the $\zeta$-function regularization of the resolvent of the Dirac operator which appears in (1), i.e.,

$$
\begin{gathered}
i \not D_{3} \lim _{s \rightarrow 1} R(x, y, ; s)=\delta^{(3)}(x-y) \\
i \not D_{3}=\left(i \frac{\partial}{\partial t}-H^{(\mu)}\right)
\end{gathered}
$$

where $H^{(\mu)}$ is given by Eq.(3). Explicitly we have

$$
R(x, y, ; s)=\bigvee_{k} \frac{\hat{\psi}_{k}(x) \hat{\psi}^{\dagger}(y)}{\lambda_{k}^{s}}=\operatorname{Tr}\left\langle x\left|\frac{1}{[\not D]^{s}}\right| y\right\rangle
$$

where $i \not D_{3} \hat{\psi}_{k}=\lambda_{k} \hat{\psi}_{k}$, and the sum, which runs over the full 2+1-dimensional spectrum, has been indicated formally as a pointwise trace in the last step, which in the $x \rightarrow y$ limit we shall henceforth denote with $\operatorname{Tr}$, for short.

We may now compute the curl of the regulated vacuum expectation value of the current $(7)$ :

$$
\left\langle\epsilon^{i j} \partial_{i} J^{j}\right\rangle=\lim _{s \rightarrow 1} \operatorname{Tr}\left[\alpha^{j} \epsilon^{i j} \partial_{i} \frac{1}{\left[i \frac{\partial}{\partial t}-(H+\mu)\right]^{s}}\right] .
$$

In order to determine the functional trace in Eq.(10) we diagonalize the Dirac operator in the adiabatic approximation in terms of the eigenfunctions of the Hamiltonian $H$, by compactifying time over a period $T$, which we shall let eventually to infinity in the zero-temperature limit, and introducing antiperiodic boundary conditions over $T$. 122 The eigenfunctions and eigenvalues of $i D_{3}(8)$ are then given by

$$
\begin{aligned}
& i \not D_{3} \hat{\psi}_{n k}=\lambda_{n k} \hat{\psi}_{n k} \\
& \lambda_{n k}=\frac{(2 k+1) \pi}{T}-\left(\alpha_{n}+\mu\right) \\
& \hat{\psi}_{n k}(\vec{x}, t)=\frac{1}{T} e^{i \lambda_{n k} \frac{t}{T}} \psi_{n}(\vec{x})
\end{aligned}
$$


in terms of the so-called Floquet indices (12) $\alpha_{n}$ of the instantaneous eigenfunctions and eigenvalues of the Hamiltonian (3):

$$
\begin{gathered}
H(\vec{x} ; t) \psi_{n}(\vec{x}, t)=E_{n}(t) \psi_{n}(\vec{x}, t) \\
\alpha_{n}=\frac{1}{T} \int_{0}^{T} d t E_{n}(t) .
\end{gathered}
$$

Of course this is the exact spectrum in the case of time-independent gauge fields, when $\alpha_{n}=E_{n}$.

Substituting Eq.s(11),(12) in the expression (10) for the curl of the current we get

$$
\left\langle\epsilon^{i j} \partial_{i} J^{j}\right\rangle=-\lim _{s \rightarrow 1} \partial_{i} \bigvee_{n} \sum_{k=-\infty}^{\infty} \operatorname{tr} i \alpha^{i} \gamma_{5} \frac{\frac{1}{T} \psi_{n}(x) \psi_{n}^{\dagger}(x)}{\left[\frac{(2 k+1) \pi}{T}+\alpha_{n}+\mu\right]^{s}},
$$

whence, in the particular case of time-independent fields,

$$
\begin{aligned}
\left\langle\epsilon^{i j} \partial_{i} J^{j}\right\rangle & =\lim _{s \rightarrow 1} y_{k, n} 2 E_{n} \operatorname{tr} \frac{\psi_{n}^{\dagger} \gamma_{5}(x) \psi_{n}(x)}{\left[\frac{(2 k+1) \pi}{T}+E_{n}+\mu\right]^{s}} \\
& =2 \lim _{s \rightarrow 1} \operatorname{Tr} \gamma_{5} H(D+\mu+H)^{-s},
\end{aligned}
$$

where in the last step $D=-i \frac{\partial}{\partial t}, \gamma_{5}=\sigma_{3}$, and we have reverted to the functional trace notation which now indicates both summation over the eigenfunctions $\frac{(2 k+1) \pi}{T}$ of $D$ and summation and integration over the full spectrum of the Hamiltonian $H$, Eq.(3). It is easy to see that Eq.(14) holds in general in the time-dependent case (at leading adiabatic order) for the time-average of the curl, provided the eigenvalues $E_{n}$ are replaced everywhere by the Floquet indices $\alpha_{n}(12)$.

Once the trace over eigenfrequencies of the Dirac operator $i \not_{3}$ has been reduced to the spectra of the operators $D$ and $H$ according to Eq. (14), we may proceed to compute the latter. We shall make first use of the fact that both the operators $H$ and $D$ have an antisymmetric spectrum, as proven by the existence of an operator which anticommutes with each of them, namely $\gamma_{5}$ and the timereversal operator $\mathcal{T}$, respectively: $\left\{\gamma_{5}, H\right\}=\{\mathcal{T}, D\}=0$. Using the antisymmetry of $D$ first, we get

$$
\left\langle\epsilon^{i j} \partial_{i} J^{j}\right\rangle=2 \lim _{s \rightarrow 1} \operatorname{Tr} \gamma_{5} H(\mu+H)\left[(\mu+H)^{2}-D^{2}\right]^{-s} .
$$

We may now separate the functional traces over $D$ and $H$ by taking a Mellin transform, whereby Eq.(15) becomes

$$
\left\langle\epsilon^{i j} \partial_{i} J^{j}\right\rangle=-2 \lim _{s \rightarrow 1} \int \frac{d t}{\Gamma(s)} t^{s-1} \operatorname{Tr}_{D}\left[e^{-t D^{2}}\right] \operatorname{Tr}_{H}\left[\gamma_{5}\left(\mu H+H^{2}\right) e^{t(\mu+H)^{2}}\right],
$$

where $\operatorname{Tr}_{D}$ and $\operatorname{Tr}_{H}$ denote the traces of the respective operators, which are to be evaluated, as usual, by Wick-rotating to Euclidean space and letting $T \rightarrow \infty$, in 
order to enforce the correct (Feynman) boundary conditions. Upon Wick rotation the spectrum of $H$, as well as the chemical potential $\mu$ become purely imaginary, thereby ensuring convergence of the $t$ integration, and allowing the Mellin transform that has lead to Eq.(16), at least if $s$ is kept large enough.

The trace over $D$ may now be computed explicitly in the large $T$ limit:

$$
\begin{aligned}
\lim _{T \rightarrow \infty} \operatorname{Tr}_{D}\left[e^{-t D^{2}}\right]= & \frac{1}{2 \pi} \int_{-\infty}^{\infty} d k e^{-t k^{2}} \\
= & \frac{1}{2 \sqrt{\pi t}}
\end{aligned}
$$

Using this result in Eq.(16) and inverting the Mellin transform we get

$$
\left\langle\epsilon^{i j} \partial_{i} J^{j}\right\rangle=-\lim _{s \rightarrow 1} \operatorname{Tr}_{H} \gamma_{5}\left(\mu H+H^{2}\right)[|\mu+H|]^{-s} .
$$

Before computing the trace over the spectrum of the Hamiltonian we may further simplify Eq.(18), using the antisymmetry of the spectrum of $H$ :

$$
\left\langle\epsilon^{i j} \partial_{i} J^{j}\right\rangle=\lim _{s \rightarrow 0} \widetilde{\operatorname{Tr}} \gamma_{5}|H|\left[H^{2}-\mu^{2}\right]^{-s},
$$

where $\widetilde{T r}$ denotes the trace over all eigenfrequencies of $H$ such that the respective eigenvalue $\lambda$ satisfies $\lambda>\mu$ (i.e., such that the denominator in Eq.(19) is positive definite).

The functional trace may now be computed explicitly, observing that in position space its diagonal matrix element is given by

$$
\begin{aligned}
\lim _{s \rightarrow 0}\left\langle x\left|\left[\widetilde{\operatorname{Tr}} \gamma_{5}|H|\left[H^{2}-\mu^{2}\right]^{-s}\right]\right| x\right\rangle & = \\
& =\lim _{s \rightarrow 0} \operatorname{tr} \int_{|\mu|}^{\infty} \frac{d k}{2 \pi} k \sqrt{k^{2}+\gamma_{5} B(x)}\left[k^{2}+\gamma_{5} B(x)-\mu^{2}\right]^{-s} \\
& =-\frac{1}{2 \pi}|\mu| B(x)+O\left(\left[\frac{B}{\mu^{2}}\right]^{2}\right)
\end{aligned}
$$

where $B(x)$ is as in Eq.(6), and as in the standard functional derivation of the anomaly, 13 we used the fact that

$$
\begin{aligned}
& H^{2}=D_{i} D_{i}+\gamma_{5} B(x) \\
& D_{i}=-i \partial_{i}+A_{i}
\end{aligned}
$$

while, since the trace as $s \rightarrow 0$ is dominated by its large-momentum behavior, the spectrum of $D_{i}$ may be approximated by that of $\partial_{i}$. 14

Using the expression of the trace (20) in Eq.(19) we get finally

$$
\left\langle\epsilon^{i j} \partial_{i} J^{j}(x)\right\rangle=-\frac{1}{2 \pi}|\mu| B(x)+O\left(\left[\frac{B}{\mu^{2}}\right]^{2}\right)
$$


which is our main result, and proves our conjecture: the vacuum-expectation value of the curl of the current is anomalous, and, to first order, proportional to the magnetic field. Notice that this a purely quantum effect, in that it pertains a vacuum expectation value of the electric current, which is generated by quantum fluctuations.

If we neglect higher order corrections under the assumption that the magnetic field is not too strong, Eq. (22) is immediately recognized as the London equation, which, together with the 2+1-dimensional Maxwell equation

$$
J^{i}=\frac{1}{e^{2}} \epsilon^{i j} \partial_{j} B(x)
$$

leads to the screening equation in the plane

$$
\Delta B(x)=\frac{e^{2}|\mu|}{2 \pi} B(x)
$$

i.e., to the Meissner effect with screening length

$$
\lambda=\sqrt{\frac{2 \pi}{e^{2}|\mu|}} .
$$

Let us now discuss the physical implications of this result. If a layered physical system may be considered as effectively two-dimensional, and we take the Lagrangian (11) as the effective theory of its charged excitations, with the parameter $e^{2}$ related to the typical distance between the planes which build up the threedimensional bulk of matter, then a constant magnetic field applied to that material is screened, provided in the given region $|B|<|\mu|^{2}$. For example, for high- $T_{c}$ layered superconductors typical values are $e^{2} \sim 20 \mathrm{eV}$. With a chemical potential $\mu$ of order of a few $\mathrm{eV}$ the screening length is $\lambda \sim 10^{3} \AA$, as in ordinary superconductors. When the magnetic flux density exceeds $\mu^{2}$, the approximation leading from (22) to (24) breaks down; if we take this as an estimate of the critical flux density we get $B_{c} \sim\left(10^{3} \AA\right)^{-2}$, i.e., $B_{c} \sim 10^{3}$ gauss, as in usual superconductors.

Even though the present discussion has been given in the low-temperature limit, this assumption has been used only in the computation of the trace over Matsubara frequencies, Eq.s (14)-(16), which has been performed in the limit $T \rightarrow \infty$. It actually turns out that the first order correction to these results, which is of order $\frac{\mu}{T}$, vanishes, thus suggesting that our results persist up to fairly high temperature (notice that $\left.\mu \sim 10^{4}{ }^{\circ} \mathrm{K}\right)$. Also, even though the chemical potential has played a crucial role in our discussion, one may notice that beyond the leading adiabatic approximation (to which we have confined ourselves) the spectrum (11) also receives (12) a Berry phase contribution which enters Eq.(11) in a way formally identical to the chemical potential. This suggests that by retaining such contributions our result may be generalized to zero density.

Finally, it is interesting to observe that the induced anomalous currents which screen the magnetic field according to Eq.(22) are transverse, as those which would 
be generated by a Chern-Simons term in the effective action of the theory, a characteristic feature of the Hall effect. This suggests the possibility of applying the present results to the Hall system. These and related problems are currently under investigation.

In sum, we have proven that the electric current of fermions in $2+1$ dimensions has an anomalous curl induced by quantum fluctuations, and related to the chiral anomaly of an underlying two-dimensional Euclidean theory. This curl obeys the Meissner equation and thus it induces screening of the magnetic field. This provides a firmer footing to the observation that topological and superconducting behavior seem to be rather robust features of gauge theories in $2+1$ dimensions. It would be interesting to try to apply the present considerations to the realistic lattice systems which display high- $\mathrm{T}_{c}$ superconductivity: perhaps this approach may provide some insight in the sought-for novel mechanism which is at the origin of these phenomena.

Acknowledgement: This works is based on an idea developed in discussions with P. Sodano, and reported in Ref. 16. I thank P. Sodano for collaboration in the early stages of this work, and a critical reading of the manuscript. I also thank V. Rivelles for discussions and hospitality at the department of mathematical physics of the university of São Paulo (Brazil), where part of this work was done. 


\section{References}

[1] For a review see S. Forte, Rev. Mod. Phys 64, 193 (1992)

[2] For a review see R. Jackiw Nucl. Phys. (Proc. Suppl.) 18A, 107 (1991)

[3] See "The Quantum Hall Effect", R. E. Prange and S. M. Girvin, Eds. (Springer, Berlin, 1987)

[4] See the contribution of R. B. Laughlin in Ref.[3]

[5] See F. Wilczek, ed. "Fractional Statistics and Anyon Superconductivity" (World Scientific, Singapore, 1990)

[6] J. D. Lykken, J. Sonnenschein and N. Weiss, Phys. Rev. D42 (rap. comm.), $2161(1990)$

[7] A. N. Redlich, Phys. Rev. Lett. 52, 1983 (1984)

[8] See R. Jackiw, in S. B. Treiman, R. Jackiw, B. Zumino and E. Witten, "Current Algebra and Anomalies" (World Scientific, Singapore, 1985)

[9] See A. J. Niemi and G. W. Semenoff Phys. Rep. 135, 99 (1986); P. B. Gilkey "Invariance Theory, the Heat Equation, and the Atiyah-Singer Index Theorem" (Publish or Perish, Wilmington, De, 1984)

[10] S. W. Hawking, Comm. Math. Phys. 55, 133 (1977)

[11] Notice that no subtraction is needed in that the analytic continuation of the $\zeta$-function provides automatically both the required regularization and renormalization. It is easy to generalize to the current thus defined the standard derivation of the anomaly equation based upon Pauli-Villars regularization.

[12] See A. J. Niemi and G. W. Semenoff, Phys. Rev. Lett. 55, 927 (1985); S. Forte and P. Sodano Nuovo Cim. D11, 321 (1989) and ref. therein.

[13] L. S. Brown, R. D. Carlitz and C. Lee, Phys. Rev. D16, 417 (1977)

[14] The result (20) is obtained expanding all functions of $H^{2}-\mu^{2}$ in powers of $\frac{B-\mu^{2}}{D^{2}}$. Expanding in powers of $\frac{B}{D^{2}-\mu^{2}}$ instead would produce a spurious infrared divergence which leads to a vanishing result for Eq.(20) unless it is regulated by an infrared mass, in which case the result (20) is reproduced. It is easy to verify that the same infrared divergence would also lead to vanishing of the axial anomaly.

[15] See e.g., Y. Hosotani and S. Chakravarty, Phys. Rev. B 42, 342 (1990)

[16] S. Forte and P. Sodano, Torino University Report DFTT 35/92 (unpublished) 